\title{
Symmetries of harmonic oscillator generating the zero-point and negative energies
}

\author{
Zahid Zakir *
}

\begin{abstract}
The Hamiltonian of harmonic oscillator is symmetric under the replacement of canonically conjugate variables and a canonical transformation to ladder operators maintains this symmetry. The Hamiltonian is expressed through a symmetrized product of the ladder operators and, as a result, at quantization there arise a zeropoint energy. Therefore, for quantized fields, canonically conjugate variables of which enter into the Hamiltonian unsymmetrically, the zero-point energy could not arise. A new symmetry of harmonic oscillator is found: a wave equation and its solutions do not vary at a joint changing of signs of frequency, energy and mass of a particle. It is shown that the problem of the negative norm for negative-frequency states appears at taking positive mass at negative energy and, contrary, the problem disappears at taking the same sign of mass and energy as it is required by relativistic kinematics. In the nonrelativistic theory, considered as a limiting case of relativistic theory, the states of a particle with negative frequency, energy and mass are described consistently as evolving only backward in time and representing the states of its antiparticle with positive frequency, energy and mass evolving forward in time. For such charge-conjugation symmetric system of oscillators an extended space of states with generalized operators is constructed.
\end{abstract}

PACS: 03.65.Ge, 11.30.Er, 1130.Ly, 11.90.tt

Key words: Hamiltonian dynamics, discrete symmetries, time reversal, quantization

\section{Content}

Introduction

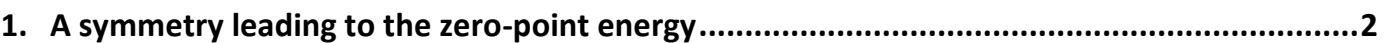

1.1. The zero-point energy for an oscillating particle ........................................................ 2

1.2. The zero-point energy for quantized fields ....................................................................

2. The extended space of states for harmonic oscillator ......................................................4

2.1. The time-reversal and the negative-frequency modes.................................................... 4

2.2. A symmetry between positive- and negative-frequency modes ........................................ 5

2.3. The extended space of states and generalized ladder operators ...................................... 7

2.4. Reinterpretation of negative-frequency states ............................................................ 9

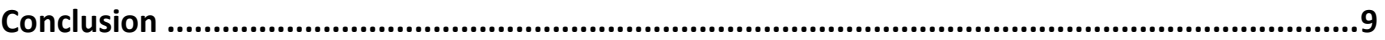

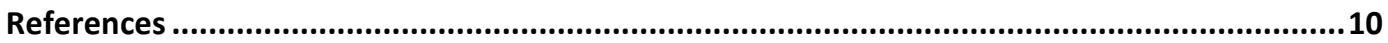

\section{Introduction}

A quantized harmonic oscillator plays a key role in modern physics since various systems, particularly free relativistic fields, are quantized by analogy with it. Two properties of the harmonic oscillator have been exploited in quantum field theory (QFT) - a formalism of ladder operators and a zero-point energy for ground states.

\footnotetext{
*Centre for Theoretical Physics and Astrophyics, Tashkent, Uzbekistan; zahidzakir@theor-phys.org
} 
However, it is wonderful that in QFT models the zero-point energy appeared due to a quite different reason than in the harmonic oscillator [1-3].

In quantum mechanics the zero-point energy of the oscillator formally follows from a symmetrized product of ladder operators and from the physical point of view it is required by the uncertainty relations. In contrast to these symmetrical and physical reasons, in QFT the zero-point energy usually appears at transition from the ladder operators for the negative-energy particles $a(-k), a^{*}(-k)$ to the operators for the positive-energy antiparticles $b(k), b^{*}(k)$, where $k=\left(\omega_{k}, \mathbf{k}\right), \quad \omega_{k}=\sqrt{\mathbf{k}^{2}+m^{2}}$.

In this connection it is interesting to understand a true origin of the zero-point energy in the quantized systems, particularly, a role of symmetry properties of the system generating or eliminating of it and how the negative-energy modes are related with the zero-point energy.

In the first section of the paper the symmetry properties of the Hamiltonians of the quantized systems leading to the zero-point energy are considered. In the second section the quantum mechanics of the harmonic oscillator in the extended space of states including the negative-frequency modes is formulated. The similar methods in application to the relativistic fields are presented in the paper [4], where their efficiency for the solution of the zero-point vacuum energy problem is demonstrated.

\section{A symmetry leading to the zero-point energy}

\subsection{The zero-point energy for an oscillating particle}

The spectrum of a particle in the harmonic oscillatory potential contains a zero-point energy due to the uncertainty relations, i.e. due to a fundamental physical reason. In the formalism of the theory this physical requirement is realized across symmetry of the Hamiltonian under interchanging of canonically conjugate variables $p$ and $x^{\prime}=m \omega x$ :

$$
H=\frac{1}{2 m}\left[p^{2}+(m \omega x)^{2}\right]=\frac{1}{2 m}\left(p^{2}+x^{\prime 2}\right) .
$$

Really, canonical transformations to ladder operators:

$$
\begin{aligned}
& a(\omega) \equiv a(\omega, m)=\frac{1}{\sqrt{2 m \omega}}(m \omega x+i p), \\
& a^{*}(\omega) \equiv a^{*}(\omega, m)=\frac{1}{\sqrt{2 m \omega}}(m \omega x-i p) .
\end{aligned}
$$

preserve that symmetry and lead to a symmetrized product of the ladder operators. Then, after a normal ordering of the symmetrized product, there appears the zeropoint energy:

$$
H=\frac{1}{2} \omega\left(a^{*} a+a a^{*}\right)=\omega\left(a^{*} a+\frac{1}{2}\right) .
$$

However, as it is known, we can consider also non-symmetrical operator orderings of canonical variables, which are equivalent at the classical level, but lead to the different quantized Hamiltonians $H_{(+)}$and $H_{(-)}$: 


$$
\begin{aligned}
& H_{(+)}=\omega a^{*} a=\frac{1}{2 m}(m \omega x-i p)(m \omega x+i p)=H-\frac{i}{2} \omega[p, x]=H-\frac{1}{2} \omega, \\
& H_{(-)}=\omega a a^{*}=\frac{1}{2 m}(m \omega x+i p)(m \omega x-i p)=H+\frac{i}{2} \omega[p, x]=H+\frac{1}{2} \omega .
\end{aligned}
$$

There both Hamiltonians are hermitian and, moreover, in $H_{(+)}$the ladder operators are normal ordered and there no a zero-point energy. But they are unsymmetrical under direct interchanging of the canonically conjugate variables. However, the required symmetry may be restored if, in addition to the interchanging of the canonical variables, we interchange two types of Hamiltonians also: $H_{( \pm)} \rightarrow H_{(\mp)}$, i.e. if we generalize the symmetry operation.

In nonrelativistic quantum mechanics there is a physical reason why we must take as a physical Hamiltonian not $H_{(+)}$, but $H=H_{(+)}+\omega / 2$, since only the last choice coincides with the uncertainty relations and experiments. But, for other oscillating systems, such as fields, we should take as a Hamiltonian $H$, when the system has the zero-point energy, or $H_{(+)}$, if the system does not contain the zeropoint energy. In any case the basic symmetries and the experiment allow one to select an admissible variant of the Hamiltonian [4].

\subsection{The zero-point energy for quantized fields}

In QFT a direct analogy between the spectrum of harmonic oscillator and field modes there is only for the Hamiltonians symmetrized under the canonical variables. As it will be shown below, the positive- and negative-frequency contributions concern to different subspaces of the total Fock space. For this reason value of observables, having a direct physical meaning in own subspaces, at formal summing on both subspaces can distort a true physical picture of the system. For example, the products $\partial_{\mu} \varphi^{*} \cdot \partial^{\mu} \varphi$ and $\partial_{\mu} \varphi \cdot \partial^{\mu} \varphi^{*}$ are inequivalent in any subspace and the scalar field's Lagrangian:

$$
L=\frac{1}{2}\left[\left(\partial_{\mu} \varphi^{*}\right)\left(\partial^{\mu} \varphi\right)+\left(\partial_{\mu} \varphi\right)\left(\partial^{\mu} \varphi^{*}\right)-m^{2}\left(\varphi^{*} \varphi+\varphi \varphi^{*}\right)\right] .
$$

leads to corresponding Hamiltonian symmetrized in both subspaces:

$$
H=\frac{1}{2} \sum_{\mathbf{k}} \omega_{k}\left[a^{*}(k) a(k)+a(k) a^{*}(k)+a^{*}(-k) a(-k)+a(-k) a^{*}(-k)\right] .
$$

After conventional replacements of the ladder operators for the negative-energy particles to the operators for positive-energy antiparticles:

$$
a(-k)=b^{*}(k), \quad a^{*}(-k)=b(k)
$$

there appears the zero point energy $H_{(0)} / 2$ in both subspaces:

$$
H=\frac{1}{2} \sum_{\mathbf{k}} \omega_{k}\left[a^{*} a+a a^{*}+b b^{*}+b^{*} b\right]=\sum_{\mathbf{k}} \omega_{k}\left(a^{*} a+b^{*} b\right)+H_{(0)} .
$$

Here the zero-point energy appeared due to the same reason as for harmonic oscillator, i.e. due to the symmetrized product of the creation-annihilation operators for particles and antiparticles. 
However, in QFT usually we deal with the standard hermitian Lagrangians which for the charged scalar field has the form:

$$
L=\left(\partial_{\mu} \varphi^{*}\right)\left(\partial^{\mu} \varphi\right)-m^{2} \varphi^{*} \varphi,
$$

and which is unsymmetrical under the direct interchanging of the fields $\varphi^{*}, \varphi$. As the result, in the Hamiltonian the creation-annihilation operators appear as normal ordered ones for both signs on energy:

$$
H=\sum_{\mathbf{k}} \omega_{k}\left[a^{*}(k) a(k)+a^{*}(-k) a(-k)\right] .
$$

Usually the zero-point energy has been derived in this expression as the result of the transition from the operators for the negative-energy particles to the operators for the positive-energy antiparticles:

$$
\begin{aligned}
& H=\sum_{\mathbf{k}} \omega_{k}\left[a^{*}(k) a(k)+b(k) b^{*}(k)\right]= \\
& =\sum_{\mathbf{k}} \omega_{k}\left[a^{*}(k) a(k)+b^{*}(k) b(k)\right]+H_{(0)}
\end{aligned}
$$

Notice, that here the zero-point energy is related by the existence of the negative-frequency modes of the field, or antiparticles, without reference to the symmetries of the Lagrangian. In the next section we shall consider how the negative-frequency modes of the harmonic oscillator they can be taken into account rigorously and physically interpreted and then we can answer to a question about a relation between the zero-point energy and the negative-frequency states.

\section{The extended space of states for harmonic oscillator}

\subsection{The time-reversal and the negative-frequency modes}

The Schrödinger equation for the wave functions $\psi(x, t)$ and $\psi^{*}(x, t)$ at representation of wave functions for stationary states in the form:

$$
\psi(x, t)=\sum_{n=0}^{\infty} \psi_{n}(x) e^{-i E_{n} t}, \quad \psi^{*}(x,-t)=\sum_{n=0}^{\infty} \psi_{n}^{*}(x) e^{i E_{n}(-t)},
$$

lead to the same equations for the stationary states:

$$
H \psi_{n}(x)=E_{n} \psi_{n}(x), \quad H \psi_{n}^{*}(x)=E_{n} \psi_{n}^{*}(x) .
$$

Their solutions, obviously, also are identical, i.e. the spatial wave functions are real: $\psi_{n}^{*}(x)=\psi_{n}(x)$.

Thus, the equations for the stationary states and their solutions do not change at the following transformations:

a) time reversal $t \rightarrow-t$ and complex conjugation:

$$
\psi^{*}(x,-t)=\psi(x, t) \text {. }
$$

But this symmetry is equivalent to two other similar symmetries:

b) $t \rightarrow t, E \rightarrow-E$ and complex conjugation: $\psi(x, t) \rightarrow \psi^{*}(x, t)$;

c) $t \rightarrow-t, E \rightarrow-E$, but no complex conjugation.

The last two symmetries have not been considered seriously because of the appearance of the negative energy states. However, a non-relativistic limit of relativistic theories contains such states which then can be transformed into the 
states of antiparticles. Therefore, the negative energy solutions should be considered for non-relativistic wave equations also.

The case of the harmonic oscillator is especially important because of the zero-point energy in QFT has been associated with the transition from the negativefrequency operators for particles to the positive-frequency ones for antiparticles. For this reason below we will consider the role of negative-frequency modes in the oscillatory spectrum also.

The negative-frequency modes of the oscillator have been studied in the early period of QFT [1-3]. However, because of the introduction of various artificial assumptions, particularly, negative probabilities, these ideas have been considered as attempts to go beyond the standard principles of quantum mechanics. Below it will be shown that such modes can be consistently described and explained without additional hypotheses.

\subsection{A symmetry between positive- and negative-frequency modes}

At quantization of fields to a backward in time propagating particle there correspond a negative-energy mode, which means that in a rest frame a negative mass also. Therefore, in the non-relativistic limit of the oscillating particle, having an antiparticle, it is necessary to include into the theory of harmonic oscillator the states with negative energy and mass also.

The oscillatory Hamiltonian (1) describes oscillations with both signs on frequency $\pm \omega$ and changes a sign only at changing of a sign on mass $m \rightarrow-m$. At the same time, the energy of the quantized oscillator:

$$
E_{n}=\omega\left(n+\frac{1}{2}\right)
$$

changes a sign only at the changing a sign on frequency $\omega \rightarrow-\omega$. But, in the case of the non-relativistic oscillator, a simple changing a sign on mass or frequency separately leads to inadmissible changing of system's properties, and many attempts in this direction have not led to the physically meaningful results.

However, this problem may be simplified and simply solved due to the existence of a new symmetry. At the combined changing of the signs on frequency, energy and mass:

$$
\omega \rightarrow-\omega, \quad E_{n} \rightarrow-E_{n}, \quad m \rightarrow-m,
$$

the wave equations and their solutions remain unchanged. Really, the wave equations for the stationary states:

$$
\frac{d^{2} \psi_{n}}{d x^{2}}+\left[2 m E_{n}-(m \omega)^{2} x^{2}\right] \psi_{n}=0,
$$

and their solutions:

$$
\psi_{n}(x)=\left(\frac{m \omega}{\pi}\right)^{1 / 4} \frac{1}{\sqrt{2^{n} n !}} e^{-\frac{1}{2} m \omega x^{2}} H_{n}(m \omega x)
$$

contain only paired products $m \omega$ and $m E_{n}$, so that at the combined changing of the signs on $m, \omega$ and $E_{n}$ they remain unchanged.

The corresponding ladder operators (2), lowering or raising the energy of the system, do not vary at the combined changing the signs on the frequency and mass: 


$$
a(-\omega,-m)=a(\omega, m), \quad a^{*}(-\omega,-m)=a^{*}(\omega, m)
$$

Therefore, the Hamiltonian for the negative-frequency modes will be exactly the same as for the positive-frequency ones, but with the opposite sign on frequency. At the quantization, from the commutation relations for the coordinate and momentum follow the commutators:

$$
\left[a( \pm \omega), a^{*}( \pm \omega)\right]=1, \quad[a( \pm \omega), a( \pm \omega)]=\left[a^{*}( \pm \omega), a^{*}( \pm \omega)\right]=0 .
$$

As the result, the Hamiltonian and the number operator $N( \pm \omega)$ take the form:

$$
\begin{aligned}
& H=\frac{1}{2} \omega\left[a^{*}(\omega) a(\omega)+a(\omega) a^{*}(\omega)\right]- \\
& -\frac{1}{2} \omega\left[a^{*}(-\omega) a(-\omega)+a(-\omega) a^{*}(-\omega)\right], \\
& N( \pm \omega)=a^{*}( \pm \omega) a( \pm \omega) .
\end{aligned}
$$

But, there appear apparent difficulties since the wave functions of these new negative-frequency levels are unorthogonal to the wave functions of the positivefrequency ones and also there appear non-zero commutators of the positivefrequency operators with the negative-frequency ones. All these are, certainly, inadmissible, since lead to the mixing of the different frequency sign states and to the instability of the system.

Nevertheless, as it will be shown below, at more adequate representation of the states the wave functions and the operators of two kind modes of oscillator in fact are orthogonal and the different frequency sign operators commutate.

For this purpose we enter the projection operators $P_{ \pm}$separating the states with the positive and negative energies accordingly:

$$
P_{+} \psi_{n}=\psi_{n(+)}, \quad P_{-} \psi_{n}=\psi_{n(-)},
$$

which have usual properties:

$$
P_{ \pm}^{2}=P_{ \pm}, \quad P_{ \pm}^{-1}=P_{ \pm}, \quad P_{+} P_{-}=P_{-} P_{+}=0 .
$$

A definite matrix representation of such projection operators will be considered in the next section, but here we will use their general properties only.

As the results of such projecting, the wave functions $\psi_{n(+)}$ and $\psi_{n(-)}$ turn out to be normalized with a positive norm and the different frequency sign wave functions turn out to be orthogonal:

$$
\int \psi_{n( \pm)}^{+} \psi_{n^{\prime}( \pm)} d x=\delta_{n n^{\prime}}, \quad \int \psi_{n( \pm)}^{+} \psi_{n^{\prime}(\mp)} d x=0,
$$

where $\psi_{n( \pm)}^{+}=\psi_{n}^{*} P_{ \pm}^{-1}$. By means of these projection operators we can define the positive and negative frequency sign ladder operators also:

$$
a_{ \pm}=P_{ \pm}^{-1} a P_{ \pm}, \quad a_{ \pm}^{+}=P_{ \pm}^{-1} a^{+} P_{ \pm} .
$$

Thus, the Hamiltonian and the number operators $N_{ \pm}$take the form:

$$
\begin{aligned}
& H=H_{+}+H_{-}=\frac{1}{2}\left\{\omega\left[a_{+}^{+} a_{+}+a_{+} a_{+}^{+}\right]-\omega\left[a_{-}^{+} a_{-}+a_{-} a_{-}^{+}\right]\right\}, \\
& N_{ \pm}=a_{ \pm}^{+} a_{ \pm} .
\end{aligned}
$$



operators:

By using (20) we obtain the commutators for the same frequency sign ladder

$$
\left[a_{ \pm}, a_{ \pm}^{+}\right]=P_{ \pm}, \quad\left[a_{ \pm}, a_{ \pm}\right]=\left[a_{ \pm}^{+}, a_{ \pm}^{+}\right]=0,
$$

and, which is very important, the vanishing commutators for the different frequency sign operators:

$$
\left[a_{ \pm}, a_{\mp}^{+}\right]=\left[a_{ \pm}^{+}, a_{\mp}^{+}\right]=\left[a_{ \pm}, a_{\mp}\right]=0 .
$$

As the result, the Hamiltonian has the form:

$$
H=\omega\left[a_{+}^{+} a_{+}+\frac{1}{2} P_{+}\right]-\omega\left[a_{-}^{+} a_{-}+\frac{1}{2} P_{-}\right] .
$$

Thus, full spectrum of the oscillator now includes not only the positive, but the negative energies also. There the zero-point energy appears in both sectors, and it is related only by the presence in the Hamiltonian (26) of the symmetrized products of the same frequency sign ladder operators.

\subsection{The extended space of states and generalized ladder operators}

Thus, for the oscillating particle there are two modes of oscillations - with positive frequency, mass and energy, and with their negative values. Since now the projected wave functions of these two modes $\psi_{n(+)}, \psi_{n^{\prime}(-)}$ are orthogonal, we can embed them into two different Fock spaces, as describing two different particles. A direct sum of these spaces then forms an extended space of states, which represents then a total space of states for the system of the oscillating particle and antiparticle in a non-relativistic limit.

The generalized wave functions of harmonic oscillator, containing both modes, we can represent in the form:

$$
\Psi_{n, n^{\prime}}^{+}=\left(\begin{array}{ll}
\psi_{n}^{*} & \psi_{n^{\prime}}^{*}
\end{array}\right), \quad \Psi_{n, n^{\prime}}=\left(\begin{array}{c}
\psi_{n} \\
\psi_{n^{\prime}}
\end{array}\right) .
$$

The projection operators then can be taken in the matrix representation:

$$
P_{+}=\left(\begin{array}{ll}
1 & 0 \\
0 & 0
\end{array}\right), \quad P_{-}=\left(\begin{array}{ll}
0 & 0 \\
0 & 1
\end{array}\right),
$$

which gives:

$$
\Psi_{n(+)}=P_{+} \Psi_{n, n^{\prime}}=\left(\begin{array}{c}
\psi_{n} \\
0
\end{array}\right), \quad \Psi_{n^{\prime}(-)}=P_{-} \Psi_{n, n^{\prime}}=\left(\begin{array}{c}
0 \\
\psi_{n^{\prime}}
\end{array}\right) .
$$

The generalized wave functions $\Psi_{n( \pm)}$ are normalized for each mode, while the functions of the different frequency sign modes are orthogonal:

$$
\begin{aligned}
& \int \Psi_{n( \pm)}^{+} \Psi_{n^{\prime}( \pm)} d x=\delta_{n n^{\prime}}, \\
& \int \Psi_{n( \pm)}^{+} \Psi_{n^{\prime}(\mp)} d x=0,
\end{aligned}
$$

Further, in the given representation the ladder operators $a_{ \pm}, a_{ \pm}^{+}$also are $2 \times 2$ matrices: 


$$
\begin{array}{ll}
a_{+}=\left(\begin{array}{cc}
a(\omega) & 0 \\
0 & 0
\end{array}\right), & a_{+}^{+}=\left(\begin{array}{cc}
a^{*}(\omega) & 0 \\
0 & 0
\end{array}\right), \\
a_{-}=\left(\begin{array}{cc}
0 & 0 \\
0 & a(-\omega)
\end{array}\right), & a_{-}^{+}=\left(\begin{array}{cc}
0 & 0 \\
0 & a^{*}(-\omega)
\end{array}\right) .
\end{array}
$$

They act on the generalized wave functions as:

$$
\begin{aligned}
& a_{+} \Psi_{n(+)}=\left(\begin{array}{ll}
a & 0 \\
0 & 0
\end{array}\right)\left(\begin{array}{c}
\psi_{n} \\
0
\end{array}\right)=\left(\begin{array}{c}
a \psi_{n} \\
0
\end{array}\right)=\left(\begin{array}{c}
\sqrt{n} \psi_{n-1} \\
0
\end{array}\right)=\sqrt{n} \Psi_{n-1,(+)}, \\
& a_{+}^{+} \Psi_{n(+)}=\sqrt{n+1} \Psi_{n+1,(+)},
\end{aligned}
$$

and also:

$$
\begin{aligned}
& a_{-} \Psi_{n^{\prime}(-)}=\left(\begin{array}{ll}
0 & 0 \\
0 & a
\end{array}\right)\left(\begin{array}{c}
0 \\
\psi_{n^{\prime}}
\end{array}\right)=\left(\begin{array}{c}
0 \\
a \psi_{n^{\prime}}
\end{array}\right)=\left(\begin{array}{c}
0 \\
\sqrt{n^{\prime}} \psi_{n^{\prime}-1}
\end{array}\right)=\sqrt{n^{\prime}} \Psi_{n^{\prime}-1,(-)}, \\
& a_{-}^{+} \Psi_{n^{\prime}(-)}=\sqrt{n^{\prime}+1} \Psi_{n^{\prime}+1,(-)} .
\end{aligned}
$$

The ground state:

$$
\Psi_{0,0}=\left(\begin{array}{c}
\psi_{0}(\omega, m) \\
\psi_{0}(-\omega,-m)
\end{array}\right) \equiv\left(\begin{array}{c}
\psi_{0(+)} \\
\psi_{0(-)}
\end{array}\right)
$$

is defined now by two relations:

$$
\begin{aligned}
& a_{+} \Psi_{0,0}=P_{+}^{-1} a P_{+} \Psi_{0,0}=\left(\begin{array}{c}
a(\omega) \psi_{0(+)} \\
0
\end{array}\right)=0, \\
& a_{-} \Psi_{0,0}=\left(\begin{array}{c}
0 \\
a(-\omega) \psi_{0(-)}
\end{array}\right)=0 .
\end{aligned}
$$

Thus, for the positive energy oscillations the ground state $\psi_{0(+)}$ is stable since the direct transitions from it to the negative energy states impossible. The new one is the same situation for the negative frequency oscillations, where the ground state $\psi_{0(-)}$ also is stable in the sense that the direct transitions from it to the positive energy states are impossible (the ladder operator $a_{-}(-\omega)$ raises the energy of a state). Moreover, now the transitions between the positive energy levels carry out only the positive energy quanta, while between the negative energy levels - only the negative energy quanta. The problems with the stability of the negative energy states appeared only if one illegally supposes that the transitions between such states occur by emitting the positive-energy quanta. If in an interaction vertex there are states of both signs of frequency, it is necessary to transfer all such states on the same sign of frequency and only after that it is possible to conclude about balance of energies in that vertex.

The Hamiltonian also has the matrix form:

$$
\tilde{H}=\omega\left(\begin{array}{cc}
a^{*}(\omega) a(\omega) & 0 \\
0 & -a^{*}(-\omega) a(-\omega)
\end{array}\right)+\frac{\omega}{2}\left(\begin{array}{cc}
1 & 0 \\
0 & -1
\end{array}\right) .
$$


The number operators $N_{ \pm}$, defined in (26), commutate with the Hamiltonian, and numbers of particles of each sign on energy conserve separately:

$$
\left[N_{ \pm}, \tilde{H}\right]=0 \text {. }
$$

Therefore, in contrast to the standard oscillator, in the system of two types of oscillators there are two conserving quantum numbers. Their combinations give us an operator of total number of quanta:

$$
N=N_{+}+N_{-}=a_{+}^{+} a_{+}+a_{-}^{+} a_{-},
$$

and an operator of the "quasi-charge" of the system:

$$
Q=N_{+}-N_{-}=a_{+}^{+} a_{+}-a_{-}^{+} a_{-} .
$$

The time reversal in this case, as it was shown above, is equivalent to the change of a sign on frequency and after that the total number of quanta $N$ does not vary, whereas the "quasi-charge" $Q$, if it is non-zero, changes a sign.

\subsection{Reinterpretation of negative-frequency states}

In the previous sections we considered the negative- and positive-frequency modes in an equal manner. It is reasonable only while we do not choose a definite direction for temporal evolution. Let this direction is chosen as a positive one when only the positive-energy states are admissible.

Then, the negative-energy states cannot be simply rejected and in the consistent nonrelativistic theory, considered as a limiting case of the relativistic theory, they should be transformed into the positive-energy states of antiparticles with the ladder operators $b_{+}$and $b_{+}^{+}$. The corresponding Hamiltonian of the system of oscillating particles and antiparticles then has the form:

$$
\begin{aligned}
& \tilde{H}=\omega\left[a_{+}^{+} a_{+}+\frac{1}{2} P_{+(a)}\right]+\omega\left[b_{+}^{+} b_{+}+\frac{1}{2} P_{+(b)}\right]= \\
& =\omega\left(\begin{array}{cc}
a^{*}(\omega) a(\omega) & 0 \\
0 & b^{*}(\omega) b(\omega)
\end{array}\right)+\frac{\omega}{2}\left(\begin{array}{ll}
1 & 0 \\
0 & 1
\end{array}\right) .
\end{aligned}
$$

If a harmonic potential acts on the particle and antiparticle similarly, i.e. the potential does not depend on a sign of charge, two kinds of oscillatory quanta in fact become indistinguishable. Under these conditions the Hamiltonian of the system has a standard form with one type quantum and with the zero-point energy:

$$
\tilde{H}=\omega\left(a^{*}(\omega) a(\omega)+\frac{1}{2}\right)\left(\begin{array}{ll}
1 & 0 \\
0 & 1
\end{array}\right) .
$$

Thus, the symmetry between the positive- and negative-frequency states of the harmonic oscillator represents in the non-relativistic limit a combined symmetry under the time-reversal and the charge conjugation.

\section{Conclusion}

Thus, we conclude that the zero-point energy formally follows only from the symmetry between the canonically-conjugate variables in the Hamiltonian and it does not depend on the existence of negative-frequency modes. Moreover, the negativefrequency modes also have the zero-point energy which after the time reversal appears as the zero-point energy of oscillating antiparticles. 
There is a new symmetry of observables and states of the harmonic oscillator under the joint changing of signs of frequency, energy and mass of an oscillating particle. There the negative mass is only (relativistic) negative energy of the particle in its rest frame. At taking into account this symmetry the norm of the negativefrequency states becomes positive.

The states of the particle with negative frequency, energy and mass should be interpreted as the states of the antiparticle with positive frequency, energy and mass. Therefore, the quantization of the harmonic oscillator by taking into account its symmetry properties allows one to describe consistently the total spectrum of states of the oscillating particle and antiparticle without any new hypothesis.

However, in the systems with a Hamiltonian without such symmetry between the canonically-conjugate variables, the direct analogy to the harmonic oscillator in the question about the zero-point energy is incorrect. It concerns the quantized fields with the standard Hamiltonians also, which are hermitian, but unsymmetrical under the interchanging of the canonically-conjugate variables. The fact that the quantized fields with the standard (non-symmetrized) Hamiltonians do not contain the zeropoint energy, will be shown in the next paper [4].

\section{References}

1. Jordan P., Pauli, W. (1928) Z. Phys., 47, 151-173.

2. Dirac P.A.M. (1942) Proc. Roy. Soc., London, A114, 243, 710.

3. Pauli W. (1943) Rev. Mod. Phys., 15, 175-207.

4. Zakir Z. (2006) Theor. Phys., Astroph.\& Cosmol., 1, 1, 11; doi: 10.9751/TPAC.2091-002 